\title{
DEGENERATIVE SPONDYLOLISTHESIS
}

\author{
J. A. W. Fitzgerald, Southampton, and P. H. Newman, London, England \\ From the Spinal Research Unit, the Institute of Orthopaedics, \\ Royal National Orthopaedic Hospital, London and Stanmore
}

\begin{abstract}
A clinical study has been made of forty-three patients with symptoms arising from degenerative spondylolisthesis of the lumbar spine. Attention is drawn to the lower average level of the iliac crests in these patients, and to the high incidence of osteoarthritis of the hips. Many patients in this series had been referred specifically for operation and fourteen were so treated. The techniques of decompression and of spinal fusion are discussed. It is concluded that patients with back pain predominant are well treated by corsetry, only a minority needing fusion, and that patients with nerve root involvement or with symptoms of spinal stenosis need decompression. The place of spinal fusion is the main problem, but it seems reasonable, firstly, in younger patients with clear evidence of instability and degenerative change at a single level, and secondly, when radical decompression is judged to increase the risk of instability.
\end{abstract}

The original description of this type of spondylolisthesis came from the examination of anatomical specimens in the Schmorl Institute by Junghanns (1930). The term "pseudospondylolisthesis" was used because of the integrity of the neural arch, both pars interarticularis and pedicle. The characteristic lesion of the pars so common in spondylolisthesis has in some clinics entitled that disorder to be termed "true spondylolisthesis". This, however, is contrary to Kilian's (1854) original meaning when he coined the word "spondylolisthesis" to describe a slowly developing displacement of a lumbar vertebra and not a lesion of the neural arch. The term "spondylolisthesis with intact neural arch" was used by Macnab (1950), but it is not the only type of spondylolisthesis with an intact neural arch, and for that reason a different term of description was sought. Because it occurs invariably similar features (Table I). This report supplements the earlier accounts of Junghanns and of Macnab. In particular the follow-up examination by one of us (J. A. W. F.) of a series of forty-three patients treated either conservatively or surgically by the other (P. H. N.) adds some features of interest to the findings and to the place of operative treatment.

\section{CLINICAL MATERIAL}

Forty-three patients who had been referred initially to a special back clinic with symptomatic degenerative spondylolisthesis were personally reviewed after intervals, from the time of first attendance, of six months to eighteen years (average six years). The patients reviewed were otherwise unselected, but the particular interest in spondy-

TABLE I

Detalls from Previous Reports

\begin{tabular}{|c|c|c|c|}
\hline & Junghanns (1930) & Macnab (1950) & $\underset{(1963)}{\text { Newman and Stone }}$ \\
\hline Total number of cases of spondylolisthesis & 60 & 142 & 319 \\
\hline Cases of degenerative spondylolisthesis & $14(23 \cdot 3$ per cent $)$ & $22(15 \cdot 5$ per cent $)$ & $80(25 \cdot 1$ per cent $)$ \\
\hline Women . & $11(78 \cdot 6$ per cent $)$ & $15(68 \cdot 2$ per cent $)$ & 58 ( 72.6 per cent $)$ \\
\hline Age range (years) & 56 to 87 & Average 60 & 40 to 91 \\
\hline
\end{tabular}

in middle age or later life as a complication of degenerative change, Newman and Stone (1963) introduced the term "degenerative spondylolisthesis".

The details given in the original reports show many lolisthesis of one of us (P. H. N.) undoubtedly increased the proportion of those who were operated upon, as a number were referred from other centres with operation in view.

Based on a paper read at the meeting of the British Orthopaedic Association in Oxford on September 10, 1971.

J. A. W. Fitzgerald, F.R.C.S., Orthopaedic and Accident Department, Southampton General Hospital, Tremona Road, Southampton, Hampshire, England.

P. H. Newman, D.S.O., M.C., F.R.C.S., Royal National Orthopaedic Hospital, Brockley Hill, Stanmore, Middlesex HA7 4LP, England. 
The clinical features were similar to those described by Junghanns in 1930. Women predominated (thirty-nine/ forty-three). The age at presentation varied from fortythree to seventy-four years (average sixty-one years). The lumbar 4-5 level was nearly always the one affected (forty/forty-three). The degree of slip was only mild, averaging 17 per cent of the antero-posterior diameter of the fifth lumbar vertebral body.

\section{Associated osteoarthritis of the hips and other joints}

A feature of the group as a whole was the high incidence of osteoarthritis of the hips, which affected seven of the forty-three patients (17 per cent) and in almost all cases was severe enough to require operation.
The relationship between the two disorders is uncertain. It may be that these patients are examples of the generalised polyarticular form of arthritis described by Kellgren and Moore (1952) and by Lawrence, de Graaf and Laine (1963). This contention is supported by the fact that in nine of the twelve hips affected (five of the seven cases were bilateral), the pattern was one of protrusio acetabuli (Fig. 1), a feature again stressed by Sharp (1969). Furthermore, in a number of these cases other joints were also affected, notably the knees, the cervical spine and the first carpo-metacarpal joints. Finally, in this particular sub-group the degenerative changes were generalised throughout the lumbar and lower thoracic spine, affecting both zygapophysial joints

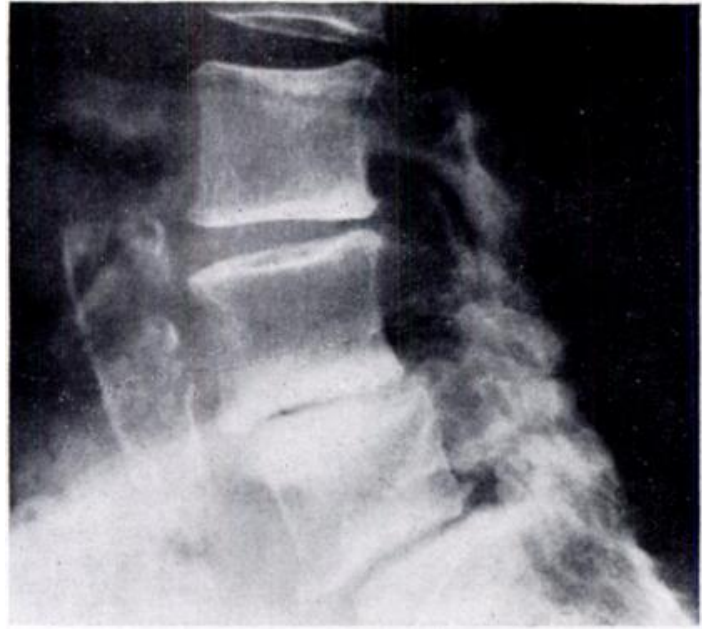

Radiographs of a woman aged 65 showing the generalised changes in the zygapophysial joints and discs throughout the lumbar spine commonly seen in association with osteoarthritis of the hips. Note also the calcification of the aorta.
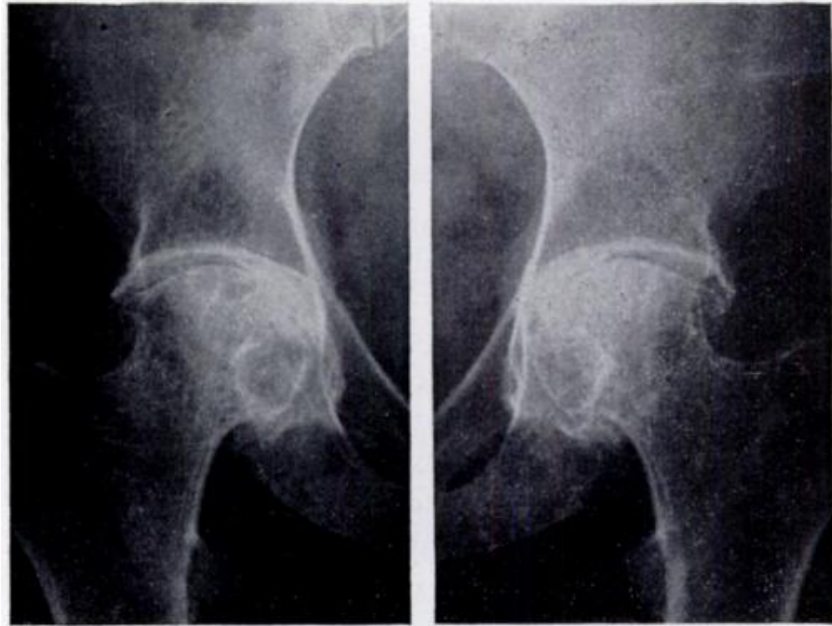

FiG. 1

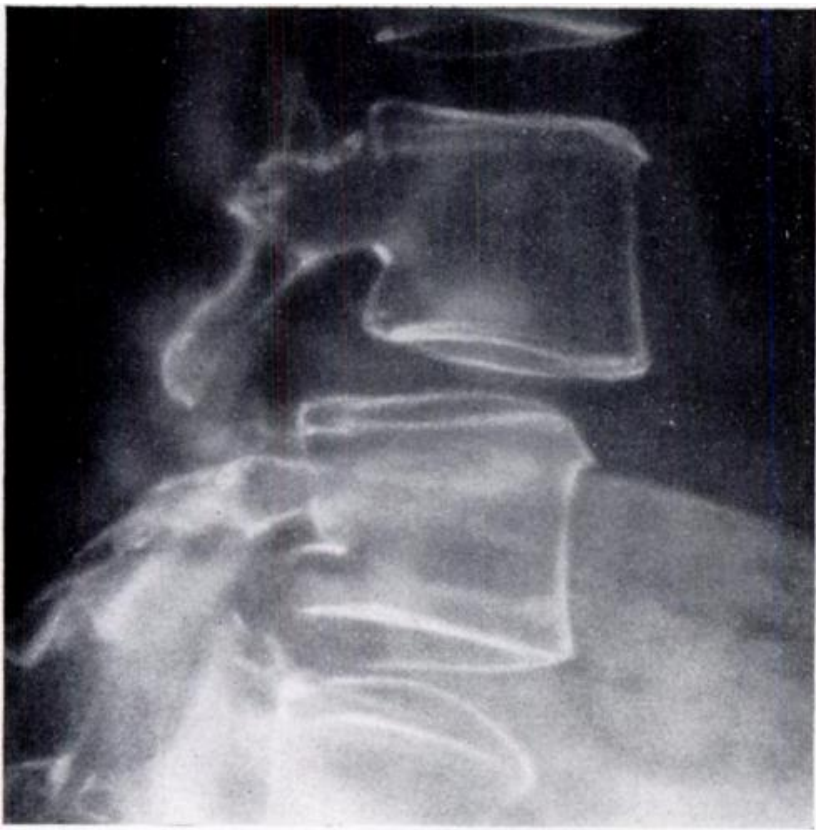

FIG. 2

A lateral radiograph of a typical case of degenerative spondylolisthesis. The level of the iliac crests is seen to be below the L.4-5 disc space.

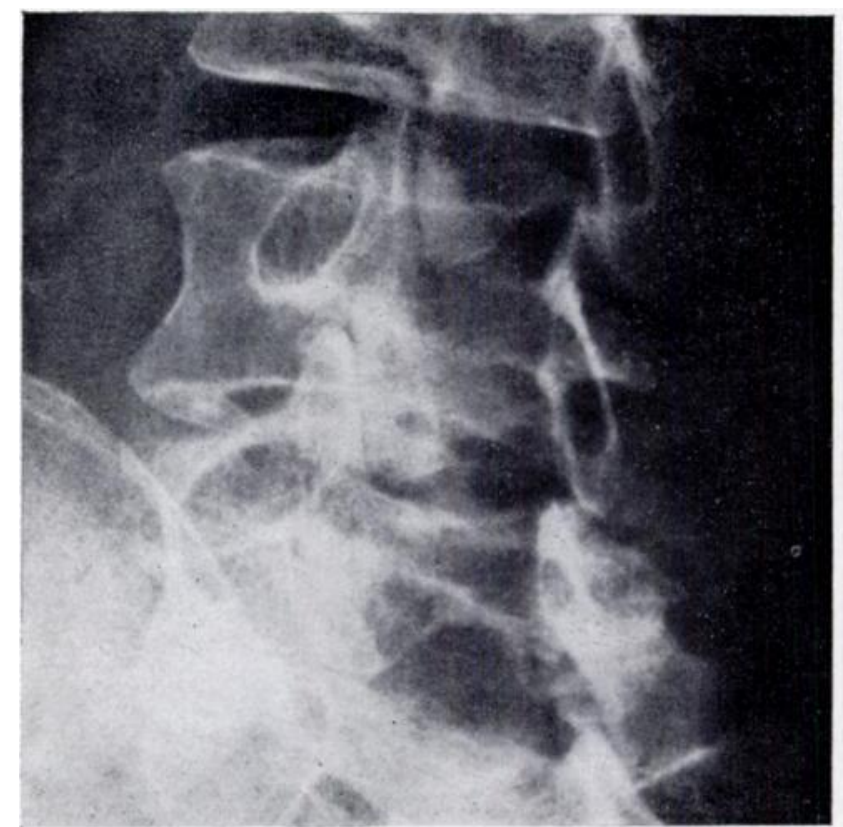

FIG. 3

Same case as in Figure 2. The oblique radiograph, which is the first to show the typical degenerative changes in the zygapophysial joints. 
and discs, whereas in the more typical cases of degenerative spondylolisthesis the zygapophysial joints alone were affected at the single level of L.4-5 (Figs. 2 and 3).

\section{PATTERNS OF PRESENTATION}

As Macnab noted, there are three basic symptom patterns (Table II). Firstly, those complaining essentially of low back pain with or without leg pain but without signs of nerve root pressure (Group A). (Macnab used the term "sciatica" but here the term "leg pain" is preferred as this pain was not a true sciatica but a vaguer, deeper, pain affecting the thigh only.) In some cases the back pain was typical of lumbar instability, in others characteristic of lumbar spondylosis, but in many not sufficiently specific to allow useful subdivision of the group. Secondly, those complaining of typical sciatica with evidence of alteration of nerve root conduction, with or without back pain (Group B). Thirdly, those with the pathognomonic picture of intermittent claudication of the cauda equina as described by Blau and Logue (1961) (Group C). (Macnab postulated cauda equina compression but had no examples in his series.)

However, it must be stressed that all patients in this series attended with symptomatic degenerative spondylolisthesis. Many cases of degenerative spondylolisthesis, perhaps the majority, are essentially symptom free, as was demonstrated in a series of cases of osteoarthritis of the hips where routine radiography of the lumbar spine revealed a number of totally unsuspected lesions.

Little difficulty was experienced in allocating each of the cases in this series to its appropriate group and seldom did a case change from one group to another.

Factors were sought which might characterise this grouping, but without success. Sex, age and duration of symptoms were not significantly different. Although the slip was always marked, averaging 20 per cent in cases with neurological involvement, some cases without such involvement showed an even greater degree of slip. The three cases with the greatest forward displacement (33, 30 and 28 per cent) had no neurological deficit. The size of the vertebral canal was measured by the technique of Jones and Thomson (1968) but only one case fell into their category of "small normal canal". It was concluded that the cause of nerve root or cauda equina compression was a combination of two changes-firstly exuberant osteophytes developing postero-laterally from the degenerative zygapophysial joints, and secondly, the narrowing gap between the inferior border of the neural arch of the slipping vertebra above and the postero-superior angle of the vertebral body below, which may carry a hypertrophic bar and thickened posterior longitudinal ligament.

\section{PATHOLOGICAL ANATOMY}

Spondylolisthesis occurs when the check mechanism resisting forward thrust, which is greatest in the lower lumbar region, is insufficient. The check is a bony hook consisting of the pedicle, pars interarticularis and inferior articular facet of the vertebra above, which hooks over the superior facet of the vertebra below. Apposition is maintained by the soft tissues. Features characteristic of this type of spondylolisthesis make it quite distinct from other pathological lesions that permit

TABLE II

Patterns of Presentation

slip. The neural arch is intact and probably of normal shape and structure (Newman and Stone 1963). Osteoarthritic changes in the zygapophysial joints are a constant and predominant feature and are the point of weakness of the bony hook mechanism. The check given by one facet lying behind the other loses its efficiency owing to the degenerative changes, and the inferior facets of the vertebra above gradually grind their way between the superior facets of the vertebra below, permitting forward displacement.

An understanding of the mechanism of evolution to this final state is made easier by a study of the different stages over a period of many years. Latent segmental instability, demonstrated in flexion and extension radiographs, is more common in women, particularly at the

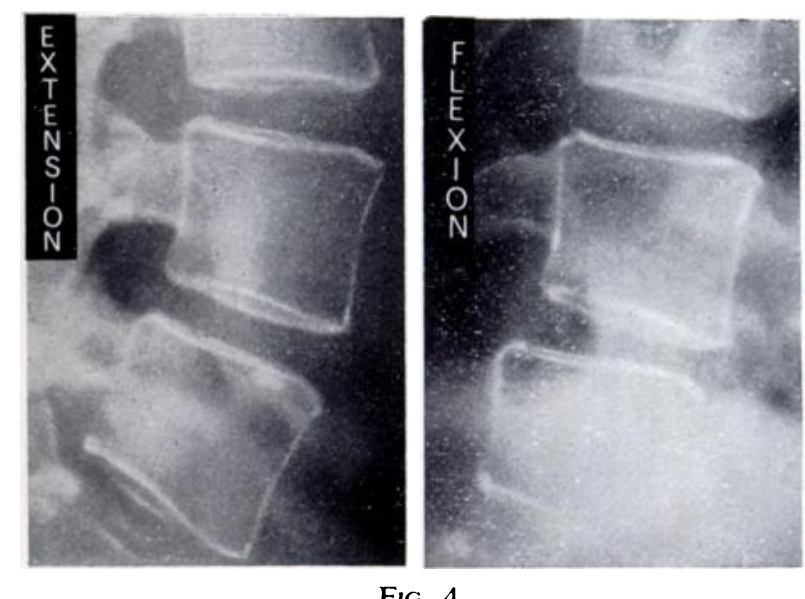

Details of lateral radiographs taken in extension and in flexion showing instability at the L.4-5 level.

lumbar 4-5 level (Fig. 4). Allbrook (1957) has shown that the fourth lumbar vertebra is the most mobile. Whereas the fifth vertebra is supported between the iliac crests and anchored by the ilio-lumbar ligaments, the fourth is only partially protected in this way, and when 
Degenerative Spondylolisthesis

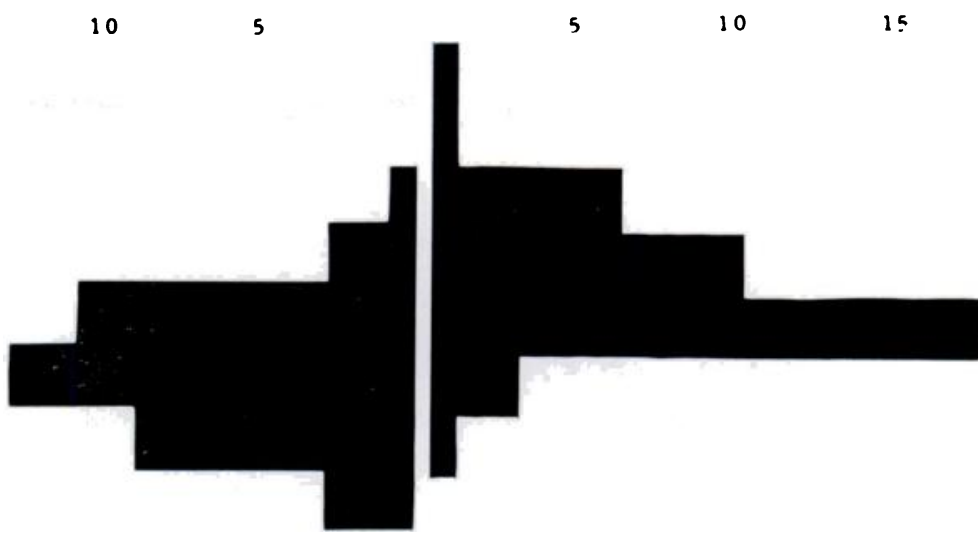

FIG. 5

A histogram of levels of the supracristal plane showing that in cases of degenerative spondylolisthesis the level is usually lower than in the normal spine.

the sacrum is relatively high it is more exposed to stress, causing instability.

Support for this hypothesis has been given by analysis of the radiographs in this series: the supracristal plane was found on average to cross the lumbar spine at a lower level than normal. A quantitative estimate of the difference was arrived at by judging in each case which quarter of the body of the fourth or fifth lumbar vertebrae was cut by the supracristal plane compared with the levels found in an equal number of apparently normal patients of similar age and sex. In the normal spine the level of the supracristal plane is most frequently at or above the lowermost quarter of the body of the fourth lumbar vertebra, whereas in degenerative spondylolisthesis it is usually at or below this level (Fig. 5).

This observation suggests that spines destined to develop this type of spondylolisthesis tend to have a congenitally high base. This reduces the protection to the fourth lumbar vertebra given by the soft tissues between the iliac crests, exposes the joint between the fourth and fifth vertebra to increased stress and leads to instability. Once instability has developed it is countered by increased tone of the sacrospinalis, giving a lordotic posture. If this remains as a chronic state an extension contracture develops, with abnormal weight stress transmitted to the neural arches and zygapophysial joints and eventual degenerative change. The lumbar 4-5 facets are set more sagittally than the lumbo-sacral facets and are therefore more prone to give way when degenerate (Fig. 6). The body of the fourth lumbar vertebra slips forward very gradually carrying with it an intact neural arch, so that the spinous process travels as far forward as the vertebral body. This is the factor which is foremost in diminishing the size of the spinal canal. The other important factor is hypertrophic change of the joints, which encroaches postero-laterally on the spinal canal and posteriorly on the lateral recesses (Fig. 7).

\section{RADIOLOGICAL FEATURES}

The essential feature is a degenerative change of the zygapophysial joints, found in every case. It is seen initially in the oblique views (Fig. 3), which also confirm the intact neural arch, but later the antero-posterior view shows that the inferior facets of the fourth lumbar vertebra are "ground in" between the superior facets of the

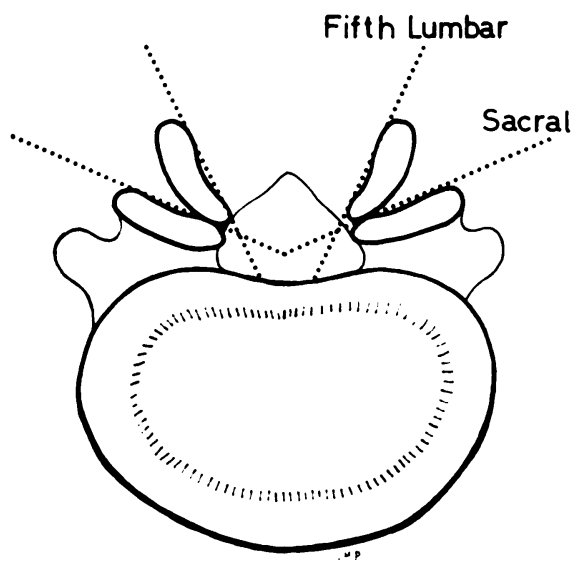

FIG. 6

A diagram to show the difference in angle between the sacral facets and the superior facets of the fifth lumbar vertebra.

fifth (Fig. 8). Lateral radiographs show the level and degree of slip and the extent of involvement of the lumbar spine. These points have been discussed above and require little further comment. The L.4-5 level alone was involved in thirty-eight cases, L.5-S.1 in two cases, and L.3-4, L.3-4 plus 4-5, and L.4-5 plus L.5-S. 1 in one case each.

The degree of slip varied from just recognisable up to 33 per cent, with an average of 17 per cent. In one patient with symptoms for only a few weeks a slip of 28 per cent was found; in another the slip increased by 12 per cent over a period of fourteen months. 
Congenital anomalies were notable for their virtual absence, being limited to partial lumbarisation or sacralisation of transitional vertebrae in four cases. Spina bifida occulta was not seen, in contrast with its frequency
Severe back pain (Group A)-Occasionally in younger subjects (forty to fifty-five years) the lesion itself gives severe back pain in the absence of sciatica and stenosis and then warrants simple stabilisation.
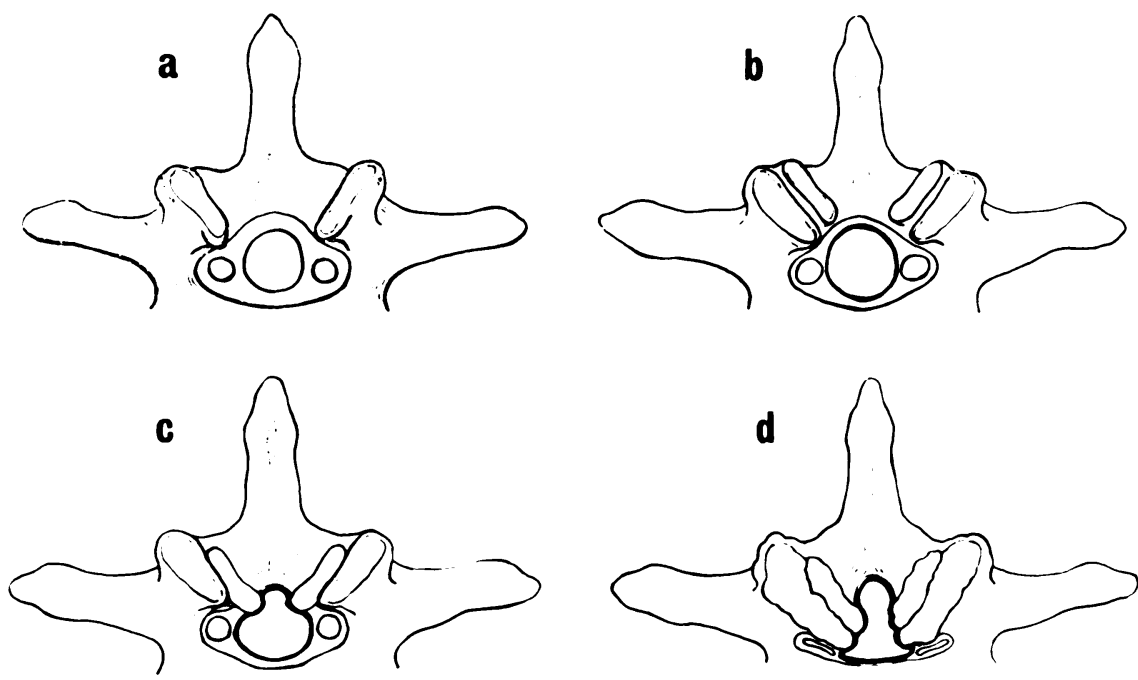

Fig. 7

Diagrams to show the compression effects of forward slip and osteophytosis on the fifth lumbar roots and theca. The outlines of the superior aspect of $L .5$ have the inferior facets of L.4 inserted in Figures b, c and d to show these effects.

in spondylolisthesis at the lumbo-sacral level. Thus Newman and Stone (1963) in a report of 131 cases of spondylolisthesis of all types at the lumbo-sacral level found an incidence of 59.5 per cent, but only 6.1 per cent in sixty-six cases at a higher level.

Myelography-This was performed in seven of the nine patients with neurological symptoms. It showed a complete or almost complete block in each case and demonstrated impingement on the theca of the zygapophysial osteophytes (Fig. 9). This investigation shows the importance of myelography in all cases with neurological signs, because a negative myelogram suggests some other cause. One patient in this series had no myelogram, and the symptoms later proved to be due to multiple sclerosis. In a similar case a normal myelogram prevented a repetition of that mistake.

\section{TREATMENT}

Of the thirty-four patients in Group A, twenty-nine were treated conservatively with lumbo-sacral corsets and five by operation. All nine patients in Groups B and C were treated surgically after conservative measures such as traction, exercise or manipulation had been tried without success.

\section{Indications for operation}

The object in most cases is decompression of the theca and nerve roots. In some cases stabilisation is also indicated, but there is no place for stabilisation without decompression in cases with neurological symptoms.
Persistent sciatica (Group B) - In this lesion at the L.4-5 level the fifth root is entrapped where it is deflected posteriorly over the hypertrophic edge of the postero-

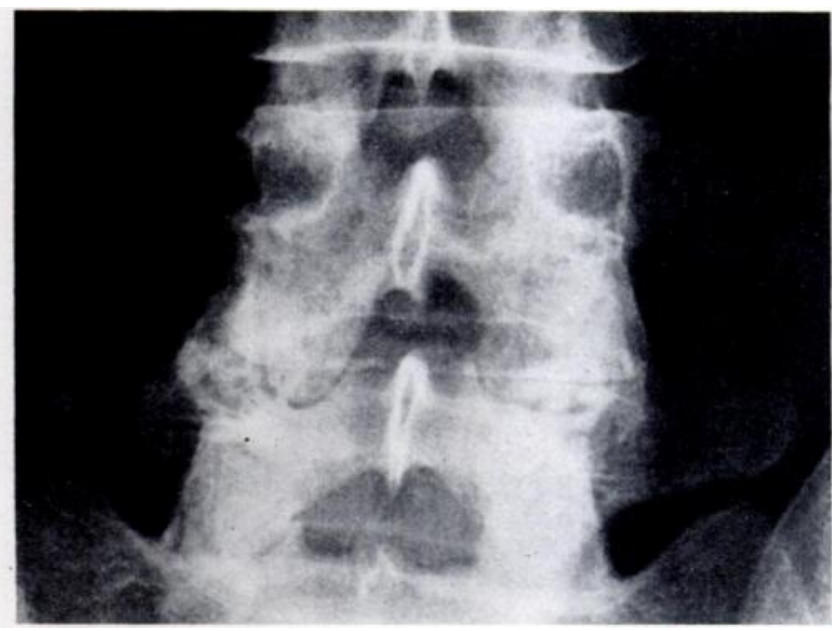

FIG. 8

A radiograph showing quite clearly on the left-hand side the "ground-in" appearance of the inferior facets of L. 4 between the superior facets of L.5, which is typical of established degenerative spondylolisthesis.

superior angle of the body of L.5, between this and the hypertrophic facets of the zygapophysial joints (Fig. 10). Spinal stenosis (Group C)-The theca is constricted at the point where it is carried forward by the advancing neural arch of L.4, held back by the hypertrophic posterosuperior edge of L.5 and encroached on postero-laterally by the two hypertrophic zygapophysial joints (Fig. 11). 

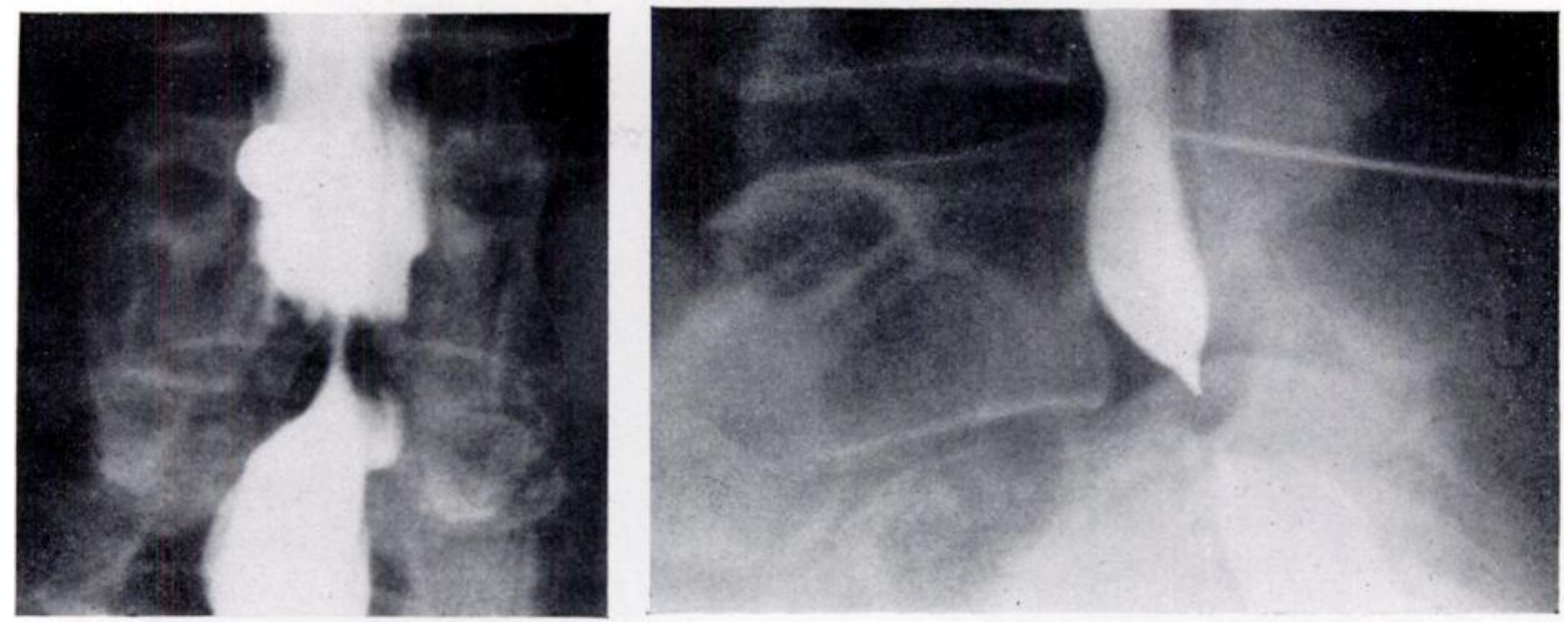

Fig. 9

Details from antero-posterior and lateral myelographs of a patient with intermittent claudication of the cauda equina and degenerative spondylolisthesis at the L.4-5 level demonstrating, on the left, postero-lateral impingement of the osteophytic zygapophysial joints, and on the right, constriction of the theca between the advancing neural arch of L.4 and the posterior angle of the body of L.S.

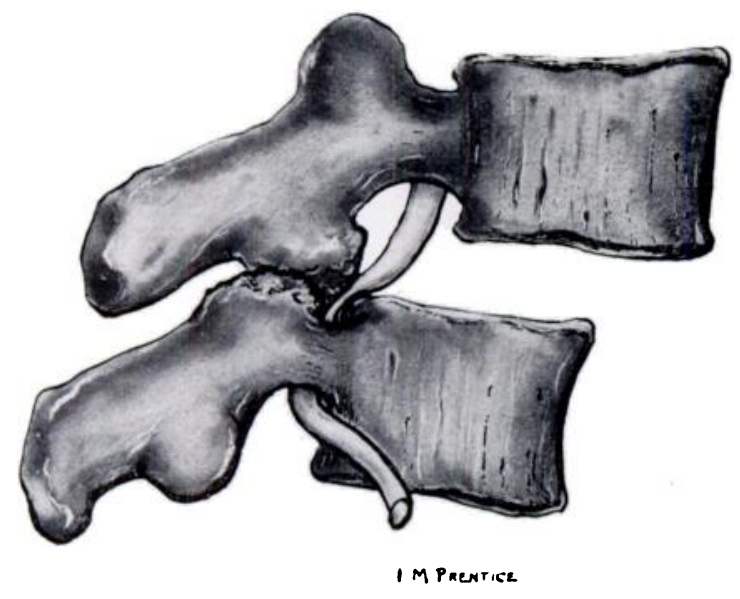

Fig. 10

A diagram to show how the fifth lumbar roots become trapped in the lateral recesses.

\section{TECHNIQUE OF OPERATION}

The patient lies prone over a pelvic support leaving the abdomen and lower chest free of pressure. The lesion is located without difficulty by the forward displacement of the spinous process of the displaced vertebra.

The zygapophysial joints present as two rugged domes of bone, in some cases overgrowing completely the ligamentum flavum. The exuberant part of both masses is removed so as to define the joint lines and expose the interlaminar space. The ligamentum flavum is excised and the medial third of each facet is either nibbled away or cut away with a small osteotome. This amounts to a bilateral double partial vertical facetectomy, which at once exposes and decompresses the entrapped nerve roots, for example, the fifth lumbar roots at the L.4-5 level (Fig. 12c).

The theca may be constricted by the lower edge of the fourth neural arch and by the two hypertrophic joints.

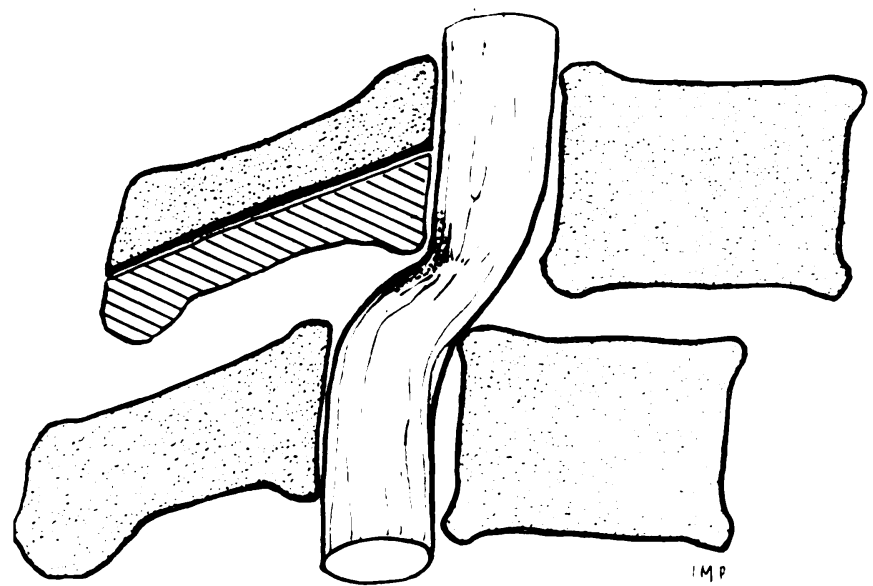

Fig. 11

A diagram to show how the neural arch of L.4 may compress the theca against the body of L.S. The shaded area indicates the resection that may be required.

The area of constriction may be white and thickened and pulsation may be absent distally (Fig. 12d).

Removal of the lower half of the fourth neural arch may be sufficient, but if there is doubt the whole arch should be excised. The theca proximal to the constriction is ballooned, and when this is seen decompression has already been achieved and pulsation should have been re-established. With both the roots and theca free of compression the primary object has been achieved and no more is necessary in most cases. In younger and more active patients fusion may be indicated because the amount of slip may increase after operation.

\section{Method of fusion}

Occasionally there is sufficient facet bone remaining to permit fixation by long screws. If not, postero-lateral grafts can be placed over the transverse processes and adjoining outer parts of the facets and pedicles. Alternatively, fusion can be promoted by splitting the spinous 


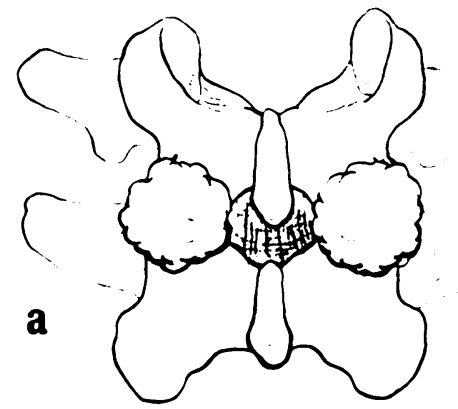

\section{L4}
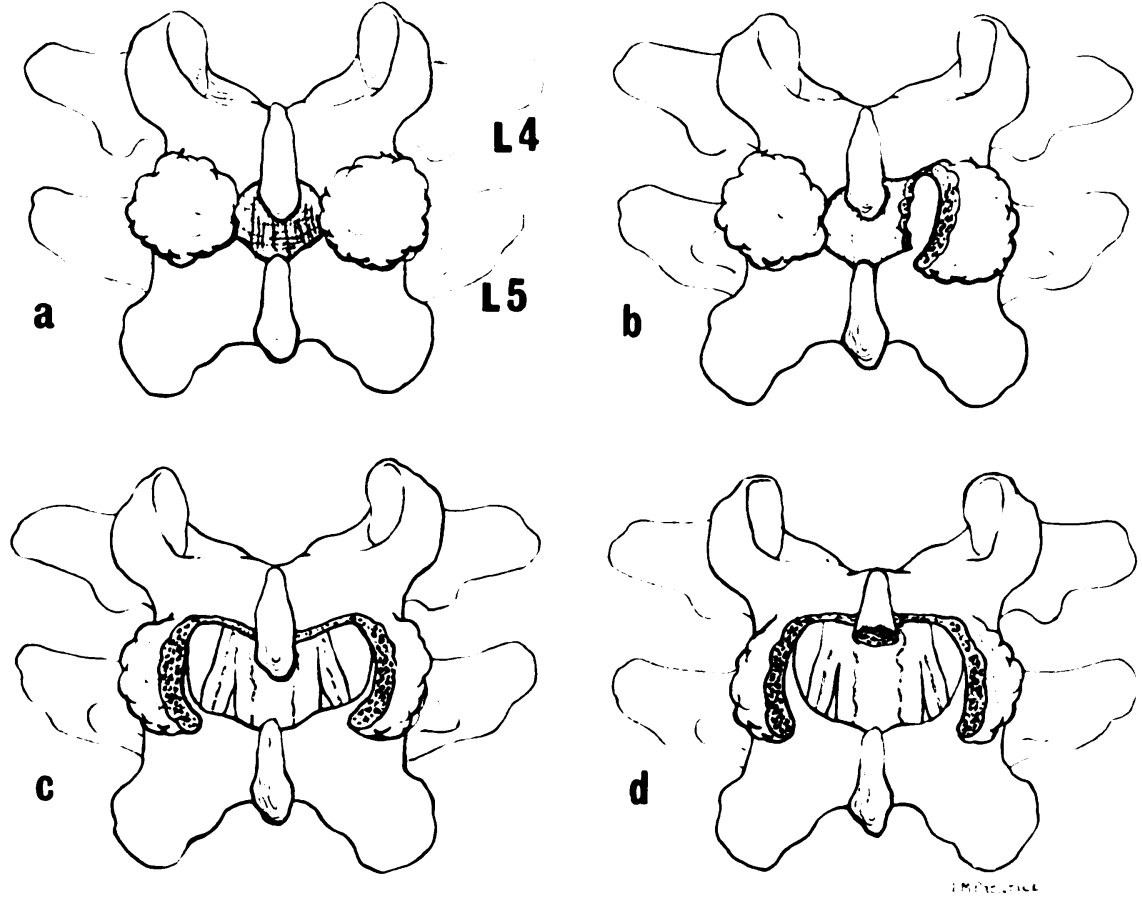

FIG. 12

Diagrams to illustrate the stages of decompression: a) Exposure of the osteoarthritic joints between $L .4$ and $L .5 ;$ b) partial resection of the inferior facet of L.4 on one side; c) bilateral double facetectomy with decompression of both fifth lumbar roots; and d) finally, partial excision of the spinous process and inferior half of the neural arch of L.4.

processes sagittally, inserting a cortico-medullary graft from the ilium and fixing it with special staples (Fig. 13). This, combined with posterior sliver grafts, gives a satisfactory fusion. Early resumption of walking may be encouraged.

\section{RESULTS}

The problem was to assess the course of a predominantly subjective condition in ageing subjects with declining functional capabilities. Success or failure therefore had to be based on the subjective assessment of the patient.

TABLE III

Type and Result of Operation in Fourteen Patients

\begin{tabular}{|c|c|c|}
\hline Group & Satisfactory & Unsatisfactory (with reason for failure) \\
\hline$\underset{\text { (back pain) }}{\mathrm{A}}$ & Decompression plus fusion L.4-S.1 & $\begin{array}{l}\text { Fusion L.3-S.1 (Instability and } \\
\text { degeneration L.2-3) }\end{array}$ \\
\hline & $\begin{array}{l}\text { Decompression plus fusion L.4-S.1 } \\
\text { Fusion L.3-S.1 }\end{array}$ & $\begin{array}{l}\text { Decompression plus fusion L.4-S.1 } \\
\text { (Instability and degeneration L.3-4) }\end{array}$ \\
\hline $\begin{array}{c}\text { B } \\
\text { (nerve root } \\
\text { compression) }\end{array}$ & $\begin{array}{l}\text { Decompression plus fusion L.4-S.1 } \\
\text { Decompression } \\
\text { Decompression plus fusion L.3-S.1 } \\
\text { Decompression plus fusion L.4-5 }\end{array}$ & $\begin{array}{l}\text { Decompression plus fusion L.4-5 } \\
\text { (Non-specific backache) }\end{array}$ \\
\hline $\begin{array}{c}\mathrm{C} \\
\text { (cauda equina } \\
\text { claudication) }\end{array}$ & $\begin{array}{l}\text { Decompression plus fusion L.4-S.1 } \\
\text { Decompression plus fusion L.4-5 } \\
\text { Decompression }\end{array}$ & $\begin{array}{l}\text { Fusion L.3-S.1 (Persistent } \\
\text { claudication) }\end{array}$ \\
\hline
\end{tabular}

Fusion after complete removal of the spinous process and neural arch is much more difficult and is seldom justified, but anterior fusion is a later possibility.
Patients were asked if they considered their overall condition was better, unchanged or worse as the result of treatment, and further whether they considered them- 


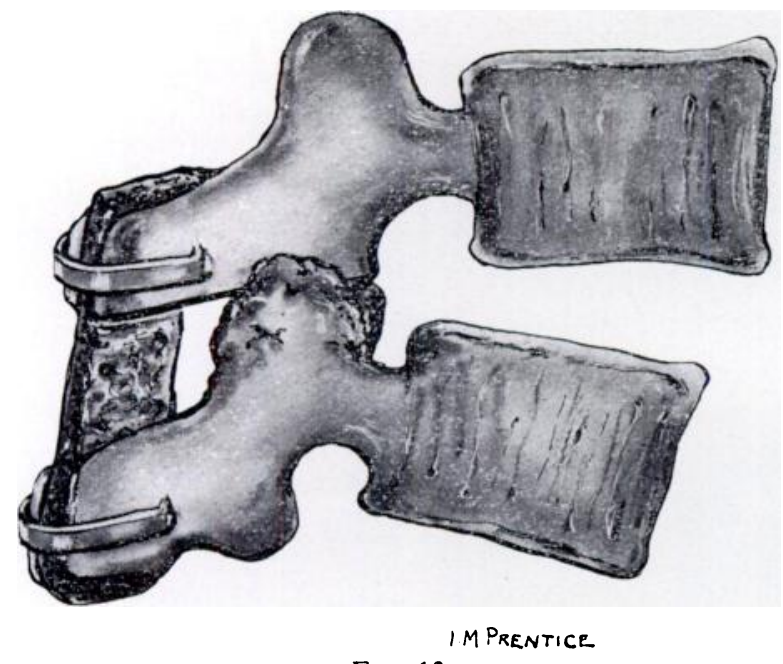

FIG. 13

Fusion of L. 4 and 5 by intraspinous graft and stapling.

selves (i) symptom free, (ii) left with minor persisting symptoms but previous activities unrestricted, (iii) with previous activities restricted, (iv) with partial improvement of some symptoms only, or (v) frankly disabled. The result of treatment was classified as satisfactory if the patient was conscious of improvement, being either symptom free or having only minor complaints. All others were classified as unsatisfactory.

\section{Conservative treatment}

In Group $A$ all but two of the twenty-nine patients

\section{Operative treatment}

Operative treatment was given in all patients in Groups $\mathrm{B}$ and $\mathrm{C}$, and in five in Group A who were younger and more active with clear symptoms and signs of instability and who demanded more definite treatment for severe back symptoms. Altogether sixteen cases were operated upon, with satisfactory results in ten. In three cases decompression alone was performed, in three fusion alone, and in ten decompression plus fusion (Table III).

An analysis of the unsatisfactory results is interesting and constructive. In one patient multiple sclerosis later became obvious. A second patient underwent decompression of the lumbar spine at L.4-5 level and bilateral McKee-Farrar total replacement for severe osteoarthritis of the hips. She was a poor historian and her moderate persisting symptoms defied analysis. These two patients are excluded from Table III and further analysis.

With the exception of one patient all neurological symptoms were relieved by operation. These were the most gratifying of the benefits conferred. For example, a man whose walking was limited to fifty yards was restored to full painless activity by decompression. In the case of the single exception intermittent claudication of the cauda equina had been treated by fusion alone; as might be expected, the symptoms persisted.

The three remaining unsatisfactory cases were relegated to that category by persisting back symptoms. In all three either fusion or decompression plus fusion had been performed, and in all three the fusion was sound.

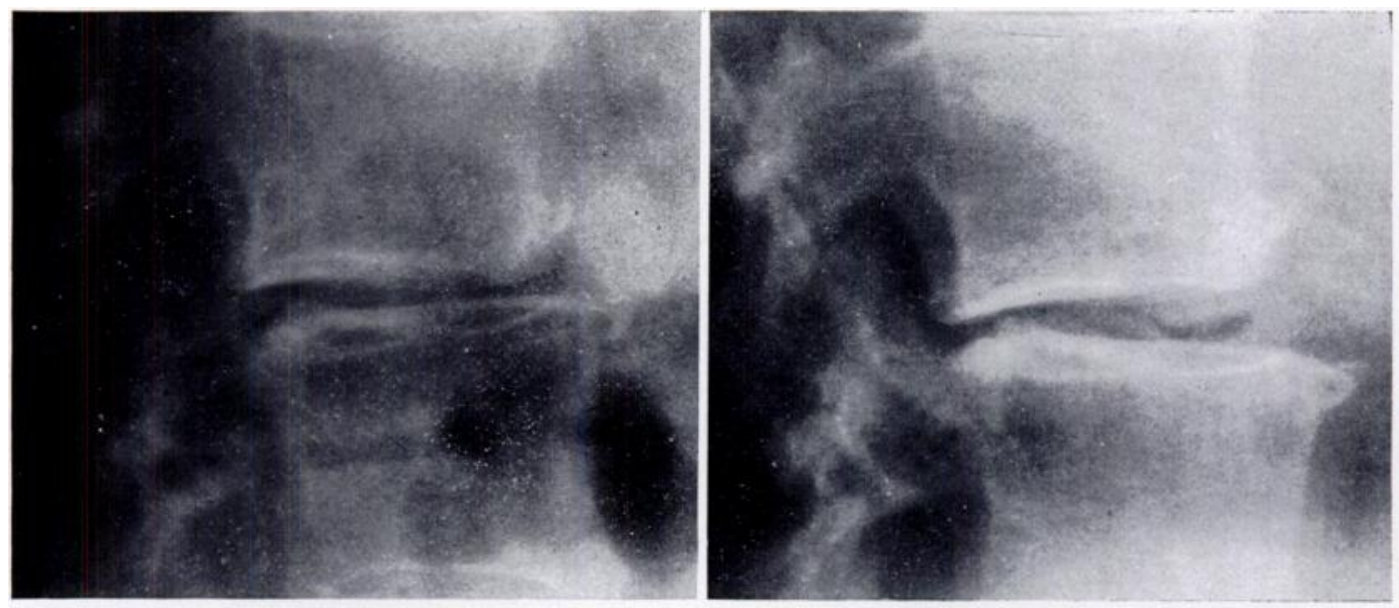

FIG. 14

Degeneration has increased and instability has developed above a spinal fusion. Left-Flexion. Right-Extension.

treated conservatively with lumbo-sacral corsets were satisfactory by the above criteria. Analgesics were seldom taken. Most striking was the regularity with which these patients returned over the years for new corsets. The two unsatisfactory results were due to problems associated with the wearing of a corset per se. For example, in one patient with a hiatus hernia the symptoms were made worse.

In Groups B and C conservative management was tried in a number of patients but without success.
In two there was obvious degeneration and instability above the fusion (Fig. 14) but in the third the cause of the persistent symptoms was uncertain.

Although not giving rise to significant symptoms in this series, increased instability and further forward slip after decompression alone did occur and is at least a potential problem. Three patients were treated by decompression alone. In one the slip did not increase and there were no symptoms of instability; in the second the 
slip did not increase but there were definite symptoms of instability; while in the third the slip increased dramatically from 20 to 40 per cent (Fig. 15), a degree not otherwise seen.

\section{CONCLUSIONS}

In this review of the treatment of degenerative spondylolisthesis at the L.4-5 level three points have become place of spinal fusion. Fusion of one segment of a spine with generalised degeneration may aggravate symptoms at a different level. On the other hand adequate decompression alone may result in instability and further slip.

Spinal fusion seems reasonable in two circumstances: firstly, in younger patients with clear symptoms and signs of instability and with degenerative change limited to a
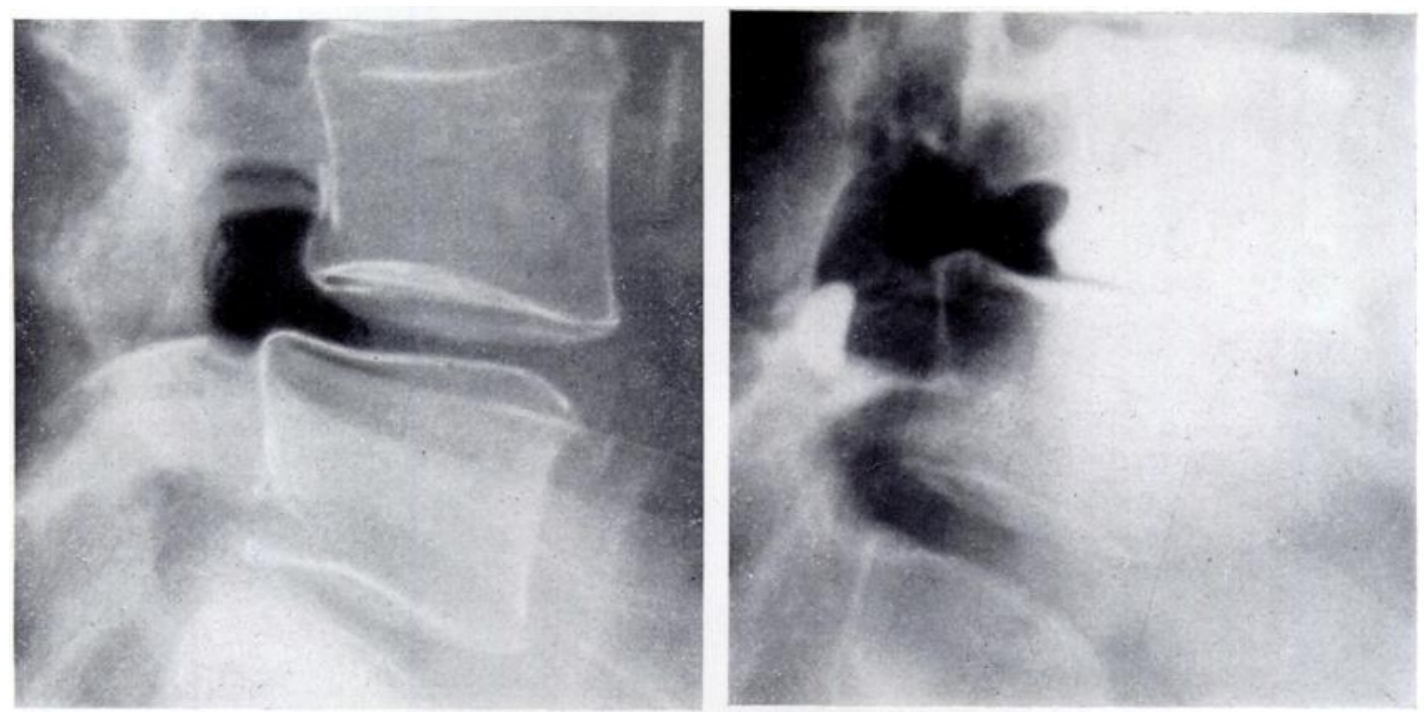

Fig. 15

In this case the degree of slip increased dramatically from 20 to 40 per cent after simple decompression. Note also that the supracristal plane is well below the level of the L.4-5 disc.

abundantly clear and provide guidance. Firstly, patients who are symptom-free need no treatment. Secondly, most patients in Group A with back pain predominant are well treated by corsetry, only a minority needing fusion. Thirdly, patients in Groups B and C who present with nerve root involvement or with symptoms of spinal stenosis need decompression. The main problem is the single level; and secondly, in some cases following decompression, again in the younger age group, particularly when adequate decompression has resulted in facet insufficiency.

Adequate decompression after myelography is essential for patients in Groups B and C, and is usually sufficient in the elderly subjects.

\section{REFERENCES}

Allbrook, D. (1957) Movements of the lumbar spinal column. Journal of Bone and Joint Surgery, 39-B, 339-345.

Blau, J. N., and Logue, V. (1961) Intermittent claudication of the cauda equina. Lancet, 1, 1081-1086.

Jones, R. A. C., and Thomson, J. L. G. (1968) The narrow lumbar canal. Journal of Bone and Joint Surgery, 50-B, 595-605.

Junghanns, H. (1930) Spondylolisthesen ohne Spalt im Zwischengelenkstück. Archiv für orthopädische und Unfall-Chirurgie, 29, $118-127$. Kellgren, J. H., and Moore, R. (1952) Generalized osteoarthritis and Heberden's nodes. British Medical Journal, 1, 181-187.

Kilian, H. F. (1854) Schilderungen neuer Beckenformen und ihres Verhaltens im Leben. Mannheim: Verlag von Bassermann and Mathy. Lawrence, J. S., de Graaf, R., and Laine, V. A. I. (1963) Degenerative joint disease in random samples and occupational groups. In The Epidemiology of Chronic Rheumatism, Volume 1, pp. 98-119. Edited by M. R. Jeffrey and J. Ball under the direction of J. H. Kellgren. Oxford: Blackwell Scientific Publications.

Macnab, I. (1950) Spondylolisthesis with an intact neural arch-the so-called pseudo-spondylolisthesis. Journal of Bone and Joint Surgery, 32-B, 325-333.

Newman, P. H., and Stone, K. H. (1963) The etiology of spondylolisthesis. Journal of Bone and Joint Surgery, 45-B, 39-59.

Sharp, J. (1969) In Textbook of the Rheumatic Diseases. Fourth edition, p. 423. Edited by W. S. C. Copeman. Edinburgh and London: E. \& S. Livingstone Ltd. 